Kunruedee Sangseethong ${ }^{a}$ Siriwanlapha Ketsilp ${ }^{b}$ Klanarong Sriroth ${ }^{\text {, c }}$

${ }^{a}$ Cassava and Starch Technology Research Unit/National Center for Genetic Engineering and Biotechnology, Bangkok, Thailand

${ }^{b}$ Department of Biotechnology, Faculty of Agro-Industry, Kasetsart University, Bangkok, Thailand

${ }^{\mathrm{C}}$ Kasetsart Agricultural and Agro-Industrial Product Improvement Institute, Kasetsart University, Bangkok, Thailand

\section{The Role of Reaction Parameters on the Preparation and Properties of Carboxymethyl Cassava Starch}

The role of reaction variables on carboxymethylation of cassava starch was investigated using a statistically experimental design approach. The reaction was carried out in an isopropanol-water mixture at $40^{\circ} \mathrm{C}$ for $3 \mathrm{~h}$. The reaction parameters under investigation included water fraction in the reaction medium, the ratio of sodium hydroxide to anhydroglucose unit (AGU) and the ratio of sodium monochloroacetate (SMCA) to AGU. The dependent responses were degree of substitution (DS) of CMS and the reaction efficiency (RE) of the carboxymethylation. Carboxymethyl cassava starches with DS in the range of 0.01 to 0.86 were prepared. The result from regression analysis indicated that the most important factors in controlling the DS of CMS were sodium hydroxide and SMCA contents followed by water content. Response surface plots suggested that the optimal levels of sodium hydroxide and water content to achieve CMS with high DS were at the intermediate values. Similar effects of sodium hydroxide and water content were also observed on the RE while SMCA content had a negative effect; increasing SMCA content resulted in lower RE. The optimal conditions to achieve the highest DS and RE were found to be at a water content of $17-19 \%$ with a molar ratio of sodium hydroxide to AGU of $1.8-1.9$ and a molar ratio of SMCA to AGU between 1.1-1.5. Carboxymethyl cassava starch with various DS was also prepared. The properties of CMS as affected by the degree of modification were also discussed.

Keywords: Carboxymethyl starch (CMS); Cassava starch; Reaction conditions; Response surface methodology; Physicochemical properties

\section{Introduction}

Carboxymethyl starch (CMS) is an important modified starch with unique properties due to the presence of negatively charged functional group $\left(\mathrm{CH}_{2} \mathrm{COO}^{-}\right)$. The introduction of carboxymethyl groups interrupts the ordered structure of native starch and intervenes with the reassociation of gelatinized starch. This modification yields starch with decreased gelatinization temperature, increased solubility and improved storage stability. Depending on the degree of substitution (DS), starch that is soluble in cold water can be produced.

CMS has a wide range of applications mainly in nonfood industries. In the pharmaceutical industry, CMS, also known as sodium starch glycolate, is commonly used as disintegrant [1]. It is also used in the textile industry as thickening agent in textile printing pastes and as sizing agent especially for hydrophilic yarn [2].

Correspondence: Klanarong Sriroth, Dept. of Biotechnology, Faculty of Agro-Industry, Kasetsart University, 50 Phahon Yothin Road, Bangkok, 10900, Thailand. Phone: +66-2-940-5634, Fax: +66-2-940-5634, e-mail: aapkrs@ku.ac.th.
CMS is produced by etherification of starch with sodium monochloroacetate (SMCA) in the presence of alkali. The first step of carboxymethylation is an alkalization where hydroxyl groups $(-\mathrm{OH})$ of starch molecules are activated and transformed into the more reactive alkoxide form (St$\left.\mathrm{O}^{-}\right)$:

$\mathrm{St}-\mathrm{OH}+\mathrm{NaOH} \rightleftarrows \mathrm{St}-\mathrm{ONa}+\mathrm{H}_{2} \mathrm{O}$

Etherification takes place in the second step:

$\mathrm{St}-\mathrm{ONa}+\mathrm{Cl}-\mathrm{CH}_{2}-\mathrm{CO}-\mathrm{ONa} \rightarrow \mathrm{St}-\mathrm{O}-\mathrm{CH}_{2}-\mathrm{COONa}+\mathrm{NaCl}$

Additionally, an undesired side reaction of SMCA with sodium hydroxide can also occur

$\mathrm{NaOH}+\mathrm{ClCH}_{2} \mathrm{COONa} \rightarrow \mathrm{HOCH}_{2} \mathrm{COONa}+\mathrm{NaCl}$

Carboxymethylation of starch can be performed using water as a reaction medium; but this process is limited to the production of CMS with degree of substitution (DS) lower than 0.07 [3]. At higher DS, the product starts to gelatinize and becomes sticky, which causes agglomeration. This prevents uniform dispersion of the reaction mixture during carboxymethylation. A process of carboxymethylation of starch paste or gelatinized starch was also 
proposed [4]. The reaction was performed in a continuous static mixer reactor optimized for concentrated aqueous starch pastes. Another method is a process in which carboxymethylation of starch is conducted in an organic solvent. The advantages of this method are that CMS with higher DS can be readily attained and the granular form of starch is preserved; thus, the side products and reagent residues can easily be washed out [5].

The properties of CMS are mainly determined by the degree of substitution (DS), i.e. the average number of carboxymethyl groups per anhydroglucose unit (AGU) [6]. Factors that influence the DS of CMS are of interest and have been investigated. The parent starch and the reaction parameters, including reagent concentration, reaction temperature, reaction time, the type of organic liquid, and water content in reaction medium, have been reported as main factors in determining the DS of CMS [5, 7-9]. Many studies reported in the literature have been conducted to determine the effect of reaction parameters on the carboxymethylation of starch by changing one variable while keeping others constant. Such approach gives no insight into the interactions between the process variables unless a large number of combinations are carried out. Statistical approach using experimental design combined with response surface methodology (RSM) can be employed to simultaneously study the influence of multiple factors on the responses of interest. The basic principle of RSM is to relate product properties (dependent variables) with processing parameters (independent variables) by means of regression equations, that describe interrelations between these variables [10]. A similar technique has recently been applied to study the modification process for arrowroot starch [11].

The objective of this study is to use the statistical approach and response surface methodology (RSM) to investigate the influence of reaction variables and their interaction on the preparation of carboxymethyl cassava starch. The reaction variables studied were the components of reaction mixture including the water content in the reaction medium, the concentration of sodium hydroxide $(\mathrm{NaOH})$ and the concentration of SMCA. The morphological and physicochemical properties of the prepared carboxymethyl cassava starch as influenced by the degree of modification were also investigated.

\section{Materials and Methods}

\subsection{Materials}

Native cassava starch was obtained from Soon Hua Seng Industry Co., Ltd., Chachoengsao, Thailand. Sodium monochloroacetate was purchased from Fluka Chemie
GmbH (Buchs, Switzerland). Isopropanol with purity higher than $99 \%$ and sodium hydroxide were obtained from BDH Limited (Poole, UK). All chemicals used in the study were of analytical grade.

\subsection{Preparation of carboxymethyl starch}

Carboxymethylation of cassava starch was carried out in an aqueous-organic liquid medium under alkaline conditions. Based on the preliminary study [12], isopropanol was the most effective organic solvent for carboxymethylation of cassava starch. Isopropanol was therefore used as organic liquid medium in this study. The reactions were carried out in a 1-L two-necked roundbottom flask equipped with a motor-driven stirrer. Starch $(10 \%, w / w)$ was dispersed in isopropanol containing various amounts of water (1.6-28.5\%, w/w, of reaction medium; the water contents already corrected for moisture present in the starch). While the slurry was vigorously stirred at $250 \mathrm{rpm}$, various amounts of $\mathrm{NaOH}$ (0.07$2.93 \mathrm{~mol} / \mathrm{mol}$ of $A G U$ ) were added. The reaction mixture was then heated to $40^{\circ} \mathrm{C}$. After stirring for $15 \mathrm{~min}$, SMCA $(0.16-1.84 \mathrm{~mol} / \mathrm{mol}$ of $\mathrm{AGU})$ was added to the mixture. The reaction was carried out for $3 \mathrm{~h}$ after which the $\mathrm{pH}$ of the reaction mixture was adjusted to 5.5-6.5 with aqueous $\mathrm{HCl}$ solution. The resulting CMS was purified by filtration and washing with $85 \%$ ethanol until the filtrate gave negative response to silver nitrate solution. The obtained starch was then dried in an oven at $40^{\circ} \mathrm{C}$.

A series of CMS with various DS (0.03-0.7) was also prepared using the optimized conditions obtained from the response surface analysis. The variation in DS was made possible by changing SMCA content in the reaction mixture. The control sample was also prepared by allowing native cassava starch undergo a treatment process exactly the same as that used for preparation of CMS but without the addition of SMCA.

\subsection{Experimental design}

A three-factor, five-level central composite design [10] was used to study the effects of reaction parameters on carboxymethylation of cassava starch. The process variables selected were: water content in the reaction medium $(1.6,7,15,23,28.5 \% \mathrm{w} / \mathrm{w}), \mathrm{NaOH}$ concentration $(0.07,0.65,1.5,2.35,2.93 \mathrm{~mol} / \mathrm{mol}$ AGU) and SMCA concentration $(0.16,0.5,1,1.5,1.84 \mathrm{~mol} / \mathrm{mol}$ AGU). The levels of each variable were coded as $-1.682,-1,0,+1$, and +1.682 for statistical analysis. The levels of each variable in this study were established according to previous reports $[5,8,12]$. Dependent variables included the $\mathrm{DS}$ of CMS and the RE of the reaction. The experimental 
design consisted of 20 experimental runs which included six replicates at the center point (Tab. 1). The experiments were conducted in randomized order to avoid systematic biases. Experimental data were statistically analyzed using Statgraphics statistical computer program (Manugistics, Inc., Rochville, USA).

\subsection{Measurement of the degree of substitution}

Degree of substitution (DS) of CMS was determined according to ISO method 11216 (1998). The carboxymethyl groups in the CMS were first converted to the acid form by acidifying with hydrochloric acid. The acidified starch was then recovered by precipitation with methanol, filtration, washing with methanol and drying in an oven. The dried starch was then treated with an excess amount of sodium hydroxide. The solution was then back-titrated with hydrochloric acid. The DS was calculated as described in the ISO method. Reaction efficiency $(R E)$ is defined as a ratio of the experimental DS to the theoretical DS $\left(D_{t}\right)$. The DSt is the maximum DS obtained for complete conversion of the limiting reactant which can be calculated as suggested by Tijsen et al. [5]:

$\mathrm{RE}(\%)=\frac{\mathrm{DS} \times 100}{\mathrm{DS} \mathrm{t}_{\mathrm{t}}}$

with

$$
\begin{aligned}
& \mathrm{DS}_{\mathrm{t}}=\frac{n_{\mathrm{SMCA}, 0}}{n_{\mathrm{AGU}, 0}} \text { if } n_{\mathrm{NaOH}, 0} \geq n_{\mathrm{SMCA}, 0} \\
& \mathrm{DS}_{\mathrm{t}}=\frac{n_{\mathrm{NaOH}, 0}}{n_{\mathrm{AGU}, 0}} \text { if } n_{\mathrm{NaOH}, 0} \leq n_{\mathrm{SMCA}, 0}
\end{aligned}
$$

\subsection{Cold water solubility}

Cold water solubility was determined in accordance with the method reported by Chen and Jane [13] with modification. A $100 \mathrm{~mL}(0.5 \%$, w/v) suspension of CMS was transferred into a blender jar and blended at low speed for $3 \mathrm{~min}$. The starch suspension was then transferred to a $250 \mathrm{~mL}$ centrifuge bottle and centrifuged at 1,200 $\times g$ for $15 \mathrm{~min}$. A $50 \mathrm{~mL}$ aliquot of supernatant was taken into a pre-weighed aluminum moisture can and dried in an air oven at $105^{\circ} \mathrm{C}$ until constant weight was obtained. Percent cold water solubility was then calculated.

\subsection{Scanning electron microscopy}

Granule morphology of starch samples was observed under JEOL scanning electron microscopy (JSM-5600, LV, Tokyo, Japan) at 10-kV acceleration and magnified at $500 \times$ and $1200 \times$.

\subsection{Viscosity measurement}

Starch $(3 \%, w / w)$ was dispersed in distilled water and left to stand for about $1 \mathrm{~h}$. The suspension was then heated in a water bath at $95^{\circ} \mathrm{C}$ for 15 min with gentle stirring in the first $5 \mathrm{~min}$. The evaporated water was added back and the solution was thoroughly mixed before the viscosity was determined. The apparent viscosity of starch paste was determined at $30^{\circ} \mathrm{C}$ using a rotational rheometer (Brookfield Engineering Laboratories, MA, USA; model DV- $\mathrm{II}^{+}$) equipped with a coaxial cylinder cell. Depending on the range of viscosity determined, spindles No. SC427 and SC4-31 were used. The sample was loaded into the sample cup, where the temperature was kept at $30^{\circ} \mathrm{C}$. The measurement was carried out after thermal equilibrium was achieved. The apparent viscosity was measured as a function of shear rate which was varied between 1.7 and $85 \mathrm{~s}^{-1}$. The readings of viscosity were taken after $30 \mathrm{~s}$ rotation at each shear rate.

\subsection{Light transmittance of starch paste}

Transmittance of starch paste was measured as described by Craig et al. [14] with some modification. A 2\% (w/ w) aqueous suspension of starch in a screw-capped test tube was heated in a boiling water bath for 30 min with constant shaking for the first $5 \mathrm{~min}$. The suspension was then cooled for $30 \mathrm{~min}$ at $25^{\circ} \mathrm{C}$. The transmittance of the fresh sample was determined against water blank with a Spectronic Genesys-5 (Milton Roy Company, New York, USA). The sample was then maintained at $4^{\circ} \mathrm{C}$ in a refrigerator and transmittance was determined after 0.25, 0.5, $1,2,3,4,6$ and 10 days of storage.

\section{Results and Discussion}

\subsection{Effects of reaction parameters on carboxymethylation}

The experimental results for DS and RE are shown in Tab. 1 and the regression coefficients for the quadratic models are given in Tab. 2. The analysis of variance (ANOVA) indicated that the models were highly significant and adequate to represent the relationship between the dependent responses (DS and RE) and the significant variables, with very small $p$-value $\left(<10^{-4}\right)$ and a satisfactory correlation coefficient $\left(R^{2} \geq 0.92\right)$.

Results from the regression analysis showed that the DS was affected mainly by the amount of $\mathrm{NaOH}$ and SMCA followed by water content. The interaction terms of SMCA with water and $\mathrm{NaOH}$ contents were also found to be significant. The positive values of the regression coeffi- 
Tab. 1. Experimental design and the responses of the dependent variables for the carboxymethylation of cassava starch.

\begin{tabular}{|c|c|c|c|c|c|c|c|c|}
\hline \multirow{3}{*}{$\begin{array}{l}\text { Run } \\
\text { no. }\end{array}$} & \multicolumn{6}{|c|}{ Independent variables } & \multirow{2}{*}{\multicolumn{2}{|c|}{$\begin{array}{l}\text { Dependent } \\
\text { variables }\end{array}$}} \\
\hline & \multicolumn{3}{|c|}{ Coded values } & \multicolumn{3}{|c|}{ Real values } & & \\
\hline & $x_{1}$ & $x_{2}$ & $x_{3}$ & $\begin{array}{l}\text { Water } \\
{[\%, w / w]}\end{array}$ & $\begin{array}{l}\mathrm{NaOH}: \mathrm{AGU} \\
{[\mathrm{mol} / \mathrm{mol}]}\end{array}$ & $\begin{array}{l}\text { SMCA:AGU } \\
{[\mathrm{mol} / \mathrm{mol}]}\end{array}$ & DS & $\begin{array}{l}\mathrm{RE} \\
{[\%]}\end{array}$ \\
\hline 1 & 0 & 0 & 0 & 15 & 1.5 & 1 & 0.71 & 71.0 \\
\hline 2 & -1.682 & 0 & 0 & 1.6 & 1.5 & 1 & 0.01 & 1.0 \\
\hline 3 & 0 & 0 & 0 & 15 & 1.5 & 1 & 0.71 & 71.0 \\
\hline 4 & 0 & 0 & -1.682 & 15 & 1.5 & 0.16 & 0.12 & 75.0 \\
\hline 5 & 0 & 0 & 1.682 & 15 & 1.5 & 1.84 & 0.86 & 57.3 \\
\hline 6 & -1 & -1 & 1 & 7 & 0.65 & 1.5 & 0.04 & 6.2 \\
\hline 7 & 0 & 0 & 0 & 15 & 1.5 & 1 & 0.70 & 70.0 \\
\hline 8 & 1 & 1 & 1 & 23 & 2.35 & 1.5 & 0.79 & 52.7 \\
\hline 9 & -1 & 1 & -1 & 7 & 2.35 & 0.5 & 0.35 & 70.0 \\
\hline 10 & 1.682 & 0 & 0 & 28.5 & 1.5 & 1 & 0.53 & 53.0 \\
\hline 11 & 1 & -1 & 1 & 23 & 0.65 & 1.5 & 0.35 & 53.9 \\
\hline 12 & 0 & 0 & 0 & 15 & 1.5 & 1 & 0.69 & 69.0 \\
\hline 13 & 0 & 1.682 & 0 & 15 & 2.93 & 1 & 0.56 & 56.0 \\
\hline 14 & 0 & -1.682 & 0 & 15 & 0.07 & 1 & 0.01 & 14.3 \\
\hline 15 & -1 & -1 & -1 & 7 & 0.65 & 0.5 & 0.09 & 18.0 \\
\hline 16 & 0 & 0 & 0 & 15 & 1.5 & 1 & 0.73 & 73.0 \\
\hline 17 & 1 & -1 & -1 & 23 & 0.65 & 0.5 & 0.17 & 34.0 \\
\hline 18 & 1 & 1 & -1 & 23 & 2.35 & 0.5 & 0.31 & 62.0 \\
\hline 19 & 0 & 0 & 0 & 15 & 1.5 & 1 & 0.72 & 72.0 \\
\hline 20 & -1 & 1 & 1 & 7 & 2.35 & 1.5 & 0.56 & 37.3 \\
\hline
\end{tabular}

Tab. 2. Regression equation coefficients for degree of substitution (DS) and reaction efficiency (RE).

\begin{tabular}{|c|c|c|c|c|}
\hline $\begin{array}{l}\text { Dependent } \\
\text { variables }\end{array}$ & $\begin{array}{l}\text { Independent } \\
\text { variables }\end{array}$ & $\begin{array}{l}\text { Coeffi- } \\
\text { cient }\end{array}$ & $\begin{array}{l}R^{2} \\
\text { (adjusted) }\end{array}$ & $\begin{array}{l}p- \\
\text { value }\end{array}$ \\
\hline DS & $\begin{array}{l}\text { Constant } \\
\mathrm{W}^{* \star *} \\
\mathrm{~N}^{\star \star \star} \\
\mathrm{S}^{\star \star \star *} \\
\mathrm{~W}^{2^{* \star \star}} \\
\mathrm{N}^{2^{* \star *}} \\
\mathrm{~S}^{2 *} \\
\mathrm{~W} \times \mathrm{S}^{\star} \\
\mathrm{N} \times \mathrm{S}^{* \star}\end{array}$ & $\begin{array}{r}0.709 \\
0.107 \\
0.167 \\
0.151 \\
-0.154 \\
-0.149 \\
-0.076 \\
0.063 \\
0.070\end{array}$ & 0.92 & $<10^{-4}$ \\
\hline $\mathrm{RE}$ & 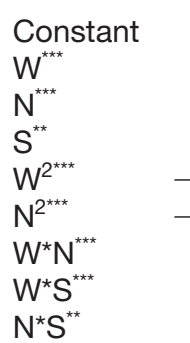 & $\begin{array}{c}70.98 \\
11.61 \\
13.19 \\
-4.67 \\
-15.41 \\
-12.53 \\
-7.045 \\
6.88 \\
-6.25\end{array}$ & 0.97 & $<10^{-4}$ \\
\hline
\end{tabular}

${ }^{* * * * * *}$, significant at $p<0.05, p<0.01, p<0.001$, respectively. $\mathrm{W}=$ water content; $\mathrm{N}=$ molar ratio of $\mathrm{NaOH}: \mathrm{AGU} ; \mathrm{S}=$ molar ratio of SMCA:AGU. cient for $\mathrm{NaOH}$ indicated that DS increased with increasing amount of $\mathrm{NaOH}$. The negative coefficient for the quadratic term suggested that, at a certain level of $\mathrm{NaOH}$, DS stopped increasing. There was an optimal value for this variable (Fig. 1a).

Similar results on the effect of $\mathrm{NaOH}$ were also observed in other studies [5, 8]. As shown in Equation (1), reaction of $\mathrm{NaOH}$ with starch results in formation of the more reactive starch alkoxide; thus, increasing the amount of $\mathrm{NaOH}$ in the reaction causes an increase in the DS. However, at higher level of $\mathrm{NaOH}$, the side reaction of $\mathrm{NaOH}$ with SMCA (Eq. 3) becomes more significant and competes with the main reaction (Eq. 2), causing a decrease in the DS [15]. As seen in Fig. 1a, the quadratic effect of $\mathrm{NaOH}$ was more pronounced at low level of SMCA where the amount of SMCA was limited. At higher SMCA content, this effect became less important due to large amount of SMCA still available for reaction with the starch molecules.

Water content posed a similar effect as $\mathrm{NaOH}$ on the DS (Fig. 1b). The DS increased with increasing water content until it reached an optimum value at intermediate level after which the DS began to decrease. An increase of DS with water content is expected because water serves as a 
(a)

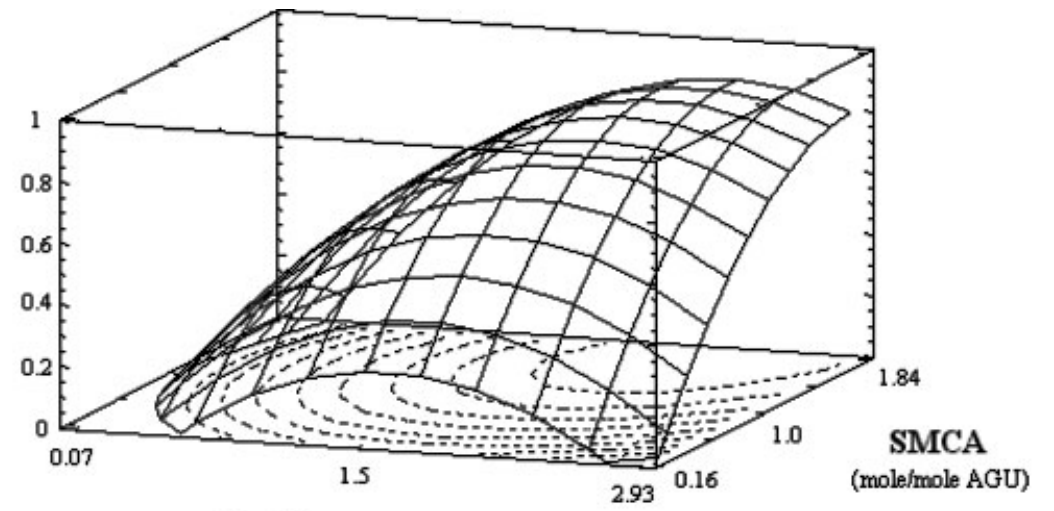

$\mathrm{NaOH}$ (mole/mole AGU)

(b)

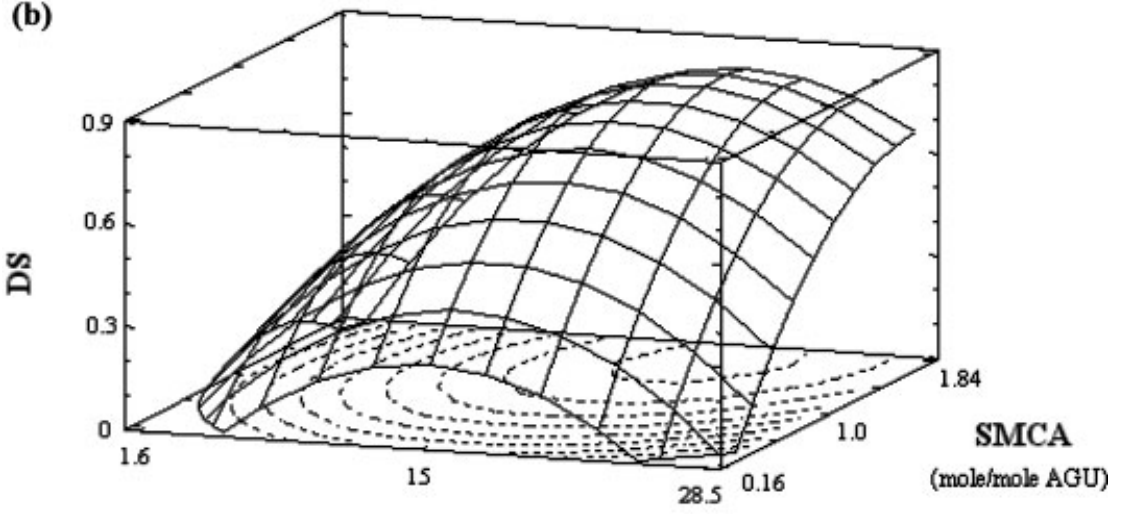

Water (\%)
Fig. 1. (a) Effects of molar ratio of $\mathrm{NaOH}$ : AGU and molar ratio of SMCA: AGU on DS of CMS (at water content of $15 \%$ ), (b) Effects of water content and molar ratio of SMCA:AGU on DS of $\mathrm{CMS}$ (at molar ratio of $\mathrm{NaOH}: \mathrm{AGU}=$ 1.5). solvent for dissolution of the reagents $(\mathrm{NaOH}$ and SMCA); furthermore, it provides a slight degree of swelling that facilitates diffusion of chemical reagents into the starch granules. A decrease in DS at the high end of water content could be explained based on the results observed by Kooijman et al. [11]. In their study, they demonstrated that at higher water fraction there was a lower selectivity of SMCA toward the main reaction (Eq. 2); while the side reaction (Eq. 3) with the formation of sodium glycolate was promoted. These could lead to the production of CMS with lower DS.

SMCA had a positive effect on the DS, especially at the high $\mathrm{NaOH}$ content. At low level of $\mathrm{NaOH}$, the amount of activated starch molecules is limited. The presence of small amounts of SMCA already saturated the available reactive sites on the starch molecules; thus, increasing amount of SMCA did not increase the DS value. As $\mathrm{NaOH}$ content increased, the effect of SMCA became prominent; the DS increased with an increase of SMCA content (Fig. 1a).
The most significant factors on RE of carboxymethylation were $\mathrm{NaOH}$ and water followed by SMCA content. $\mathrm{NaOH}$ and water content showed positive linear effect with a strong quadratic effect. These two variables gave the optimum conditions for the RE at the intermediate level (Fig. 2a). The negative value of regression coefficient indicated that SMCA inversely affected the efficiency of carboxymethylation. An increase in SMCA content resulted in a decrease in the RE, especially at high end of $\mathrm{NaOH}$ contents (Fig. 2b) This could be due to some structural change introduced to starch molecules as the reaction proceeded. The bulky carboxymethyl group added to the starch molecules could pose steric hindrance, making it more difficult for etherifying reagent to react with the remaining hydroxyl group. This effect would prevail at high SMCA concentration leading to a decrease in RE.

The strength of the models was tested by selecting conditions that were not part of the original experimental design, but in the range studied. Predicted values for both DS and RE were found to be very close to the experimental results (Tab. 3). 
(a)

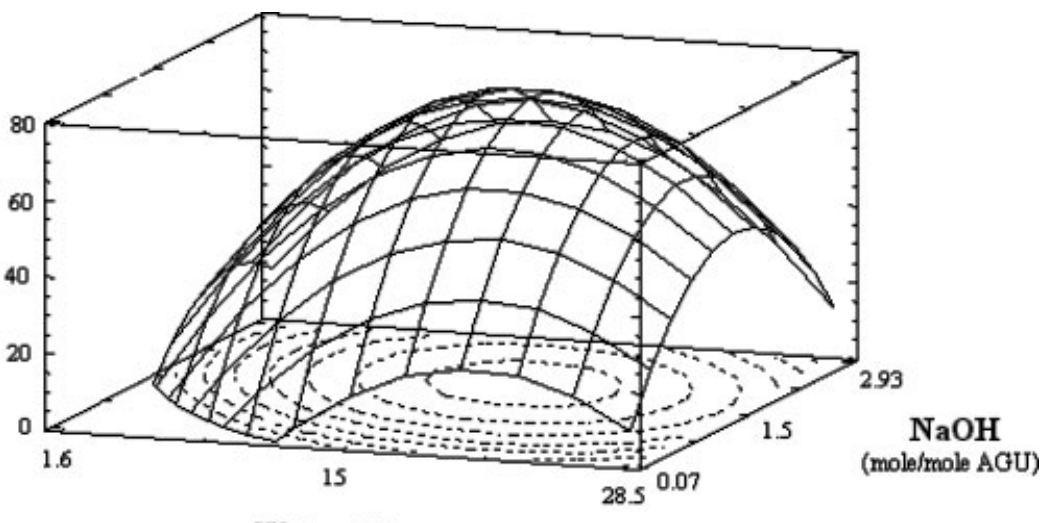

Water (\%)

(b)

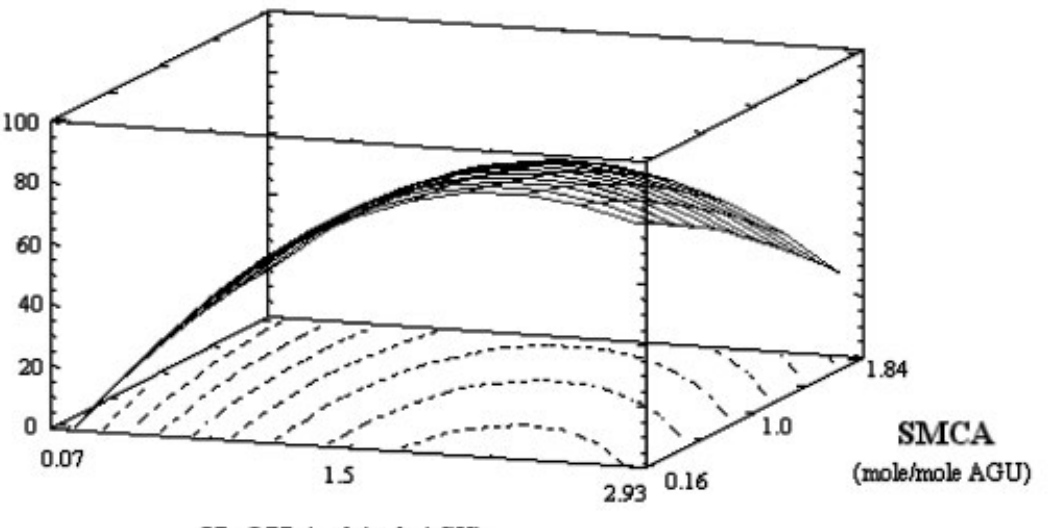

Fig. 2. (a) Effects of water content and molar ratio of $\mathrm{NaOH}: \mathrm{AGU}$ on $\mathrm{RE}$ of carboxymethylation (at molar ratio of SMCA:AGU = 1.0). (b) Effects of molar ratio of $\mathrm{NaOH}$ :AGU and molar ratio of SMCA:AGU on RE of carboxy-

$\mathrm{NaOH}$ (mole/mole AGU) methylation (at water content of 15\%).

Tab. 3. Comparison of the dependent response (DS and RE) predicted by the models with the experimental results.

\begin{tabular}{|c|c|c|c|c|c|c|}
\hline \multicolumn{3}{|c|}{ Treatments } & \multicolumn{4}{|c|}{ Responses } \\
\hline \multirow{2}{*}{$\begin{array}{l}\text { Water } \\
\left(x_{1}\right)\end{array}$} & \multirow{2}{*}{$\begin{array}{l}\mathrm{NaOH} \\
\left(x_{2}\right)\end{array}$} & \multirow{2}{*}{$\begin{array}{l}\text { SMCA } \\
\left(x_{3}\right)\end{array}$} & \multicolumn{2}{|r|}{ DS } & \multicolumn{2}{|r|}{ RE } \\
\hline & & & Predicted & Experimental & Predicted & Experimental \\
\hline 0 & 1 & 1 & 0.87 & 0.84 & 60.7 & 56.1 \\
\hline 1 & 0 & -1 & 0.37 & 0.34 & 65.0 & 68.0 \\
\hline
\end{tabular}

The conditions for the preparation of CMS with the desired DS and high reaction efficiency are of interest. These two characteristics can be used as criteria to determine optimum carboxymethylation conditions. By superimposing the contour plots of the DS and RE, the optimum conditions for production of CMS with high DS ( $>0.8$ ) while the RE is more than $70 \%$ were indicated in the shaded area in Fig. 3. The optimum compositions were found to be at a water content of $17-19 \%(w / w)$ with a molar ratio of $\mathrm{NaOH}$ :AGU of 1.8-1.9 when using a molar ratio of SMCA:AGU in the range of 1.1-1.5. These optimum conditions are in the same range of the values reported for carboxymethylation of other starches in which the levels of water are between 10 and $20 \%(\mathrm{w} / \mathrm{w})$ and $\mathrm{NaOH}$ contents are in the range of $1.5-2 \mathrm{~mol} / \mathrm{mol}$ of AGU [5, 7-9, 11].

\subsection{Properties of carboxymethyl cassava starch}

Carboxymethyl cassava starch was prepared according to the optimized conditions obtained from the previous section. By using different amounts of SMCA in the reaction mixture, the CMS with various DS (0.03-0.7) were 


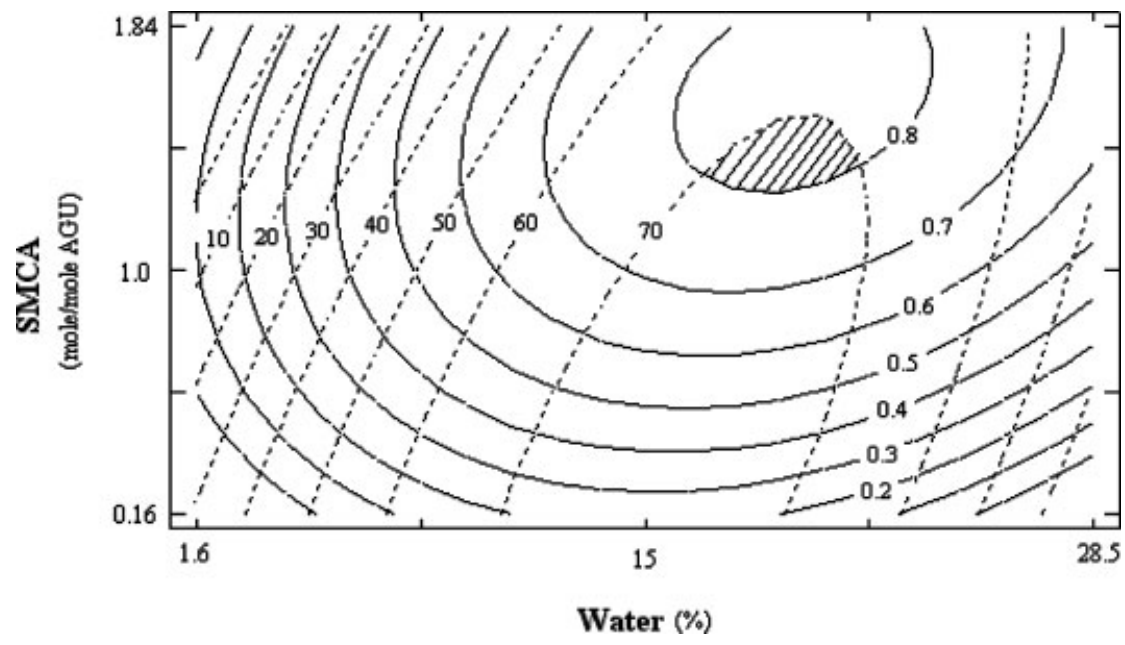

Fig. 3. Contour plots for DS (solid line) and RE (dotted line) as a function of SMCA molar ratio and water content (at molar ratio of $\mathrm{NaOH}: \mathrm{AGU}=$ 1.5). Shaded area indicates optimum conditions. obtained. A control sample was also prepared under the same conditions as those for CMS but without the addition of SMCA. The properties of the control sample were also determined along with those of the other CMS samples. This comparison allowed the separate evaluation of the effects of carboxymethylation from those of alkalization.

Tab. 4 shows the cold water solubility of starch samples. All CMS samples were completely soluble in cold water regardless of the DS. The control sample also had a very high solubility ( $90 \%)$. It seemed that an increase in cold water solubility was not only due to the introduction of negatively charged carboxymethyl groups, but also resulted from the alkalization treatment. The conditions used for the preparation of the control sample in this study were similar to the alcoholic-alkaline treatment used for preparation of granular cold water soluble (GCWS) starch [13, 16]. Chen and Jane [16] explained that the strong alkaline conditions in such treatment transformed hydroxyl groups of the starch molecules (St-OH) into alkoxide groups $\left(\mathrm{St}_{-} \mathrm{O}^{-}\right)$. The repulsion between negative charges resulted in swelling of the starch granules and exerted a tension on neighboring crystallites of starch molecules. This led to dissociation of double-helical regions and the breakup of the crystalline structure. The starch granules thus became weaker and soluble in cold water. This could have happened in the case of our control sample.

Scanning electron micrographs (SEM) of native starch, the control sample and CMS are shown in Fig. 4. Since all CMS samples had a similar appearance, only the pictures of CMS with the highest DS (0.70) are shown in this report. The native cassava starch granules were observed to be smooth, round with one truncated end. Granules of CMS and the control sample showed similar appearance, but
Tab. 4. Cold water solubility of carboxymethyl cassava starch with different DS.

\begin{tabular}{llc}
\hline & Sample & $\begin{array}{l}\text { Cold water } \\
\text { solubility [\%] }\end{array}$ \\
\hline & Native & $1.8 \pm 0.0$ \\
CMS of DS: & Control & $90.9 \pm 0.3$ \\
& 0.03 & $99.5 \pm 0.6$ \\
& 0.06 & $100 \pm 0.2$ \\
& 0.13 & $99.6 \pm 0.2$ \\
& 0.20 & $99.9 \pm 0.5$ \\
0.40 & $99.7 \pm 0.4$ \\
& 0.70 & $99.2 \pm 0.3$
\end{tabular}

markedly differed from the native starch. Although the treatment brought some changes to the granular structure of both CMS and the control sample, the integrity of the starch granules was still preserved, i.e. each granule still maintained its individual entity. Starch granules of CMS and the control sample had indented surfaces; some areas on the granule surface became shrunken and even collapsed. SEM pictures clearly revealed that the truncated end of the starch granule was more susceptible to the treatment. More indents and grooves were observed on this end of the granules. The fact that similar changes in the granular structure were detected for both CMS and the control sample indicated that the treatment during alkalization was the main factor responsible for this. The granule morphology of GCWS starches, that were prepared by an alcoholic-alkaline treatment from other studies, also displayed a similar appearance [13, 17]. It is possible that the changes observed on the starch granules were due to the loss of crystalline structure as affected by strong alkaline conditions as previously mentioned; thus, the starch granules became weaker. 


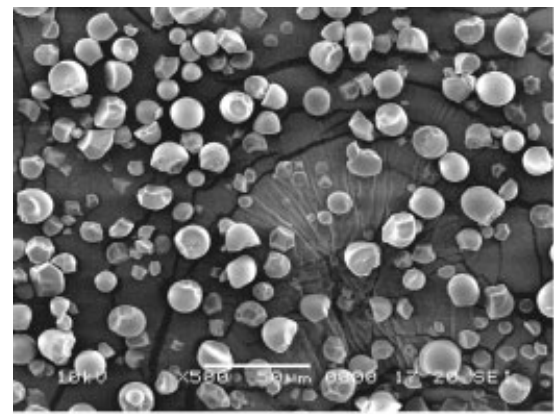

(a)

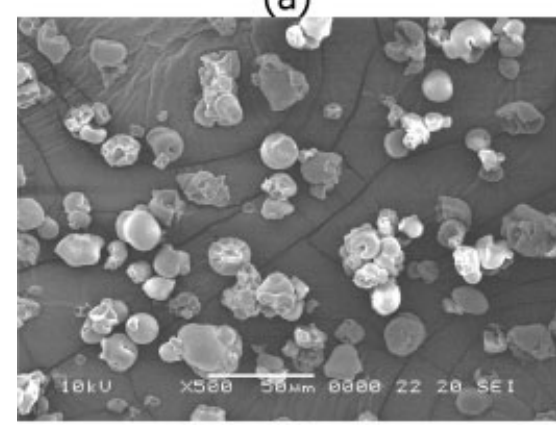

(c)

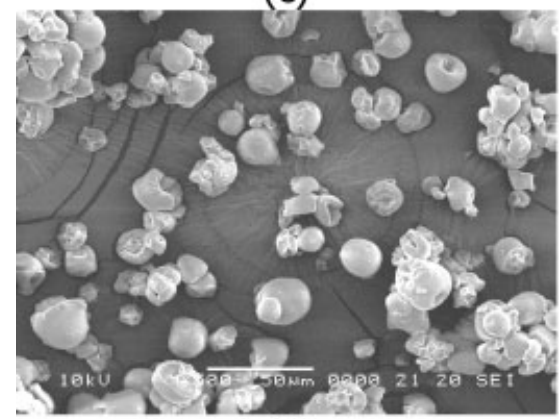

(e)

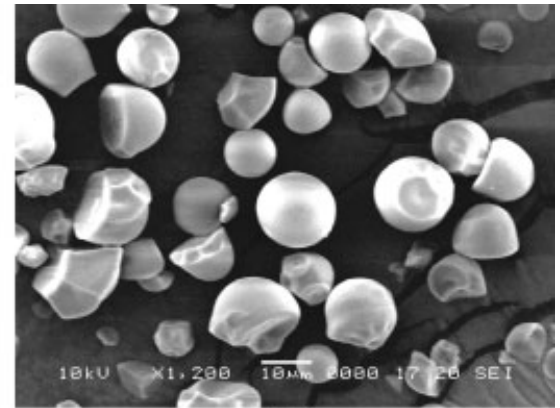

(b)

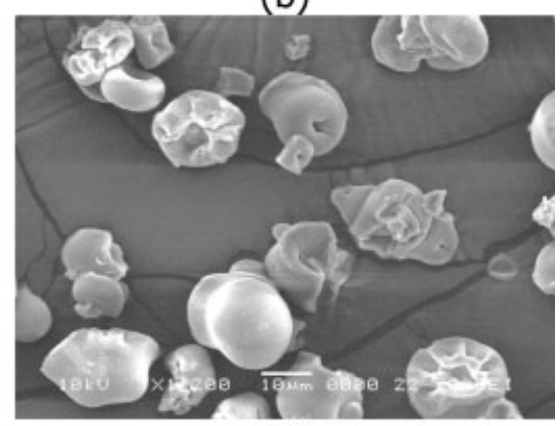

(d)

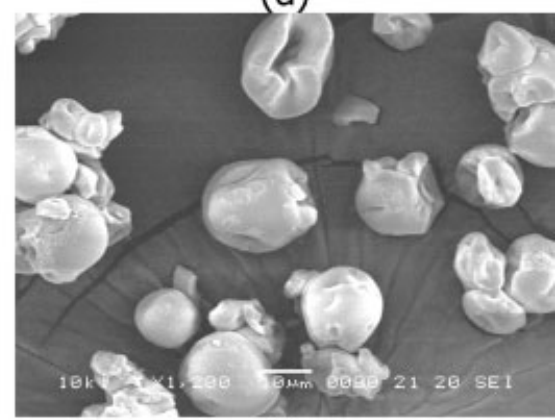

(f)
Fig. 4. Scanning electron micrographs of native cassava starch (a and b); control sample (c and d); and CMS with DS of 0.70 (e and f). a, c, e: $500 \times$; b, d, f: 1200x.
Mechanical force, i.e. stirring and grinding, that the granules experienced during preparation process could easily cause indentation on their structure.

The apparent viscosity of the starch samples as a function of shear rate is shown in Fig. 5. Native cassava starch exhibited a typical shear-thinning behavior in which the viscosity decreased with increasing shear rate. Viscosity of the control sample was substantially lower than that of the native starch. The change in viscosity of the control sample when compared to native starch was not clearly understood. It could be attributed to the structural changes caused by the strong alkaline conditions used in the preparation process. High-alkaline conditions at elevated temperature are well known to cause degradation in carbohydrate molecules. For CMS, only the samples with DS of 0.4 and 0.7 had higher viscosity than the native starch. Within the series of CMS samples, the viscosity increased with an increase in the degree of modification up to the DS of 0.4. The results demonstrated that the incorporation of carboxymethyl groups in the starch molecules improved the viscosity of starch paste compared to the control sample. The improvement of viscosity in CMS might be due to the presence of negative charges in their molecules. It is well established that viscosity of polysaccharide is a function of molecular size. Two polysaccharides with the same molecular weight but different shape or charge may display different viscosity. The molecule which requires more space for gyration will offer more resistance to flow, thus giving a higher viscosity [18]. This notion could be used to explain the phenomena observed in our study. It is common for uncharged starch molecule to form an inter- or intra-molecular association, leading to a molecule of a smaller size (smaller radius of gyration). This could be the case for the control sample. In CMS, due to the repulsion of negatively charged carboxy- 


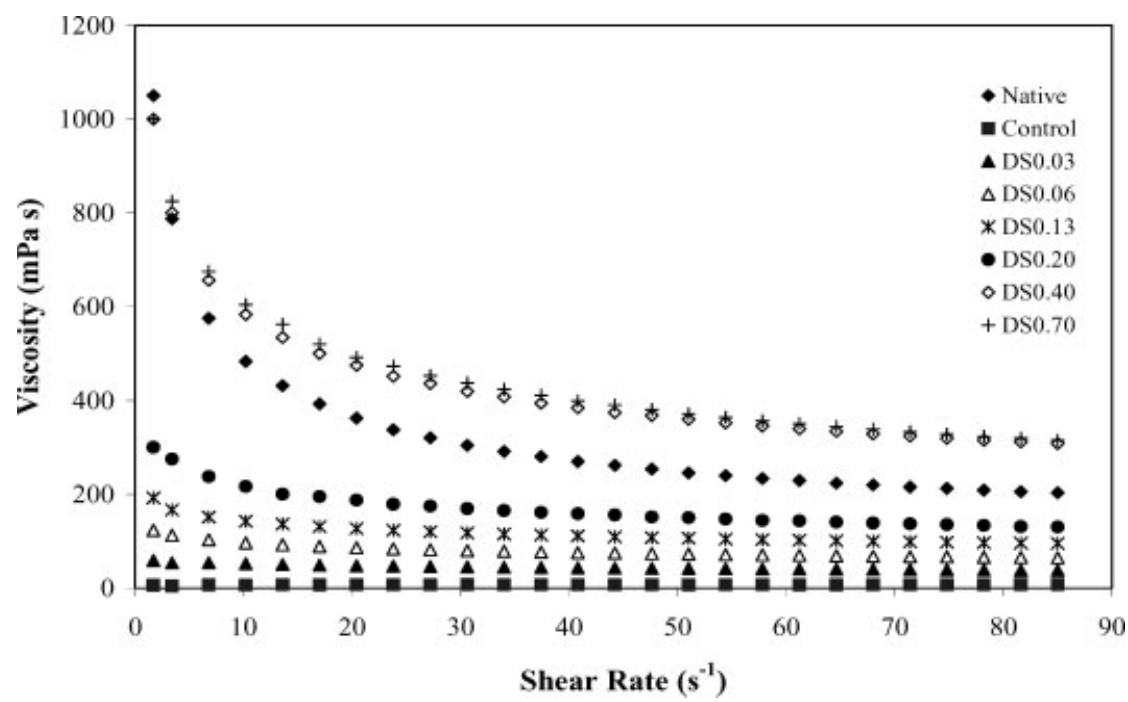

Fig. 5. Apparent viscosity of native cassava starch, the control sample and CMS as a function of shear rate. methyl groups, starch molecules tended to exist in a more expanded state; thus occupying more space for gyration and exhibiting higher viscosity. A similar trend has been observed for carboxymethyl cellulose (CMC) where viscosity rises with an increasing DS up to a DS of 0.95 [19]. Clasen and Kulicke [19] explained that this could be attributed to a change in the solution structure of cellulose derivatives. At low DS values, the compact microcrystalline components of unsubstituted cellulose still prevail. As the DS increases, the proportion of solvated molecules with higher hydrodynamic volume rises, resulting in an increase in viscosity. This is in accordance with the results observed for CMS in this study.

Light transmittance of starch pastes as a function of storage time is shown in Fig. 6. Native starch paste had an intermediate initial light transmittance (67\%) which remained constant in the first $12 \mathrm{~h}$ after which it decreased rapidly to $6 \%$ after two days of storage at $4{ }^{\circ} \mathrm{C}$. This change in paste clarity reflected the retrogradation process [20]. The control sample had an initial light transmittance lower than the native starch (45\%). This might be due to some structural changes that occurred to starch molecules during alkalization treatment. Light transmittance in the control sample dropped rapidly within the first $6 \mathrm{~h}$ to $10 \%$ and after $24 \mathrm{~h}$ the value further decreased to $2 \%$. The result from the light transmittance implied that structural changes brought about by the alkalization treatment also caused acceleration in the retrogradation process. On the other hand, all CMS samples displayed very high initial paste clarity ( $\geq 90 \%$ transmittance). The disparity of paste clarity between CMS and native starch was caused by different degree of molecular association of starch molecules in the pastes. The introduction of carboxymethyl group, even at the lowest DS, could effectively interfere with this associa- tion. Except for the lowest $\mathrm{DS}(\mathrm{DS}=0.03$ ), paste clarity of all other CMS did not decrease with storage time indicating that carboxymethyl group in the modified starch could prevent the formation of an ordered structure of the starch paste; thus, retarding retrogradation.

\section{Conclusion}

Results from the multi-linear regression models and response surface analysis provided a fairly comprehensive picture of the roles of reaction components on the carboxymethylation of cassava starch. $\mathrm{NaOH}$ was found to be the most important factor in determining the DS of $\mathrm{CMS}$ and the RE of the reaction. Increasing molar ratio of $\mathrm{NaOH}$ to AGU increased the DS and RE; but only to a certain extent. Higher amount of $\mathrm{NaOH}$ caused a decrease in both of these values. Water showed a similar effect as $\mathrm{NaOH}$ on the DS and RE. SMCA gave positive correlation to the DS; but it had a negative influence on the RE. Increasing SMCA content resulted in a decrease in the RE. The optimum conditions to obtain CMS with high DS (>0.8) and reasonable RE (>70\%) were found to be at a water content of $17-19 \%$ with a molar ratio of $\mathrm{NaOH}$ to $\mathrm{AGU}$ of $1.8-1.9$ when using a molar ratio of SMCA to AGU of 1.1-1.5. Properties of carboxymethyl cassava starch with various DS prepared under the optimized conditions were also determined. It was found that all CMS samples were completely soluble in cold water. Granular morphology of CMS displayed indentation and shrinkage on the surface. Results on the properties of the control sample clearly indicated that alkalization treatment was partly responsible for the changes in water solubility and granular structure of the modified starch. CMS showed improvement in viscosity when compared 


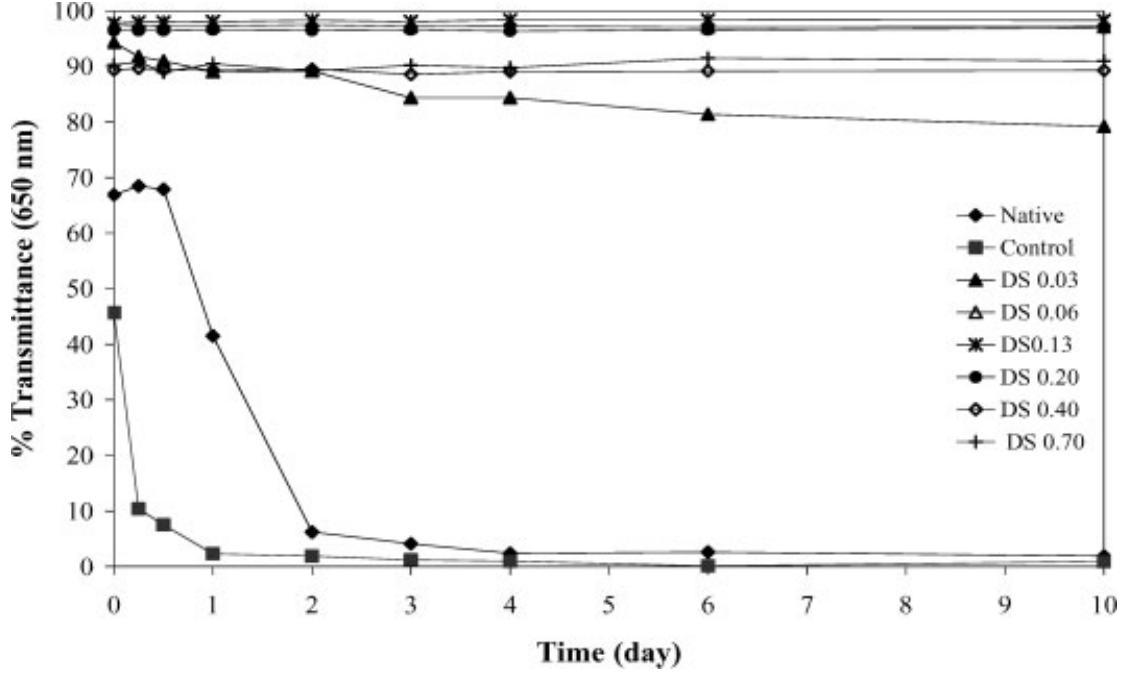

Fig. 6. Light transmittance of different starch pastes during storage at $4^{\circ} \mathrm{C}$. (Temperature of measurement $=$ $\left.25^{\circ} \mathrm{C}\right)$. to the control sample. Introduction of carboxymethyl groups into starch molecules effectively retarded the retrogradation process; CMS samples exhibited very high paste clarity even after storage at $4^{\circ} \mathrm{C}$ for 10 days.

\section{Acknowledgement}

This work was supported by National Center for Genetic Engineering and Biotechnology (BIOTEC), Ministry of Science and Technology, Thailand.

\section{References}

[1] U. Shah, L. Augsburger: Multiple sources of sodium starch glycolate, NF: evaluation of functional equivalence and development of standard performance tests. Pharm. Develop. Technol. 2002, 7, 345-359.

[2] A. A. Ragheb, H. S. El-Sayiad, A. Hebeish: Preparation and characterization of carboxymethyl starch (CMS) products and their utilization in textile printing. Starch/Stärke 1997, 49, 238-245.

[3] B. T. Hofreiter: Miscellaneous modifications, in Modified Starches: Properties and Uses (Ed. O. B. Wurzburg) CRC Press, Boca Raton, FI., 1986.

[4] A. A. C. M. Beenackers, C. J. Tijsen, J. E. Visser, E. J. Stamhuis: Novel processes for the carboxymethylation of starch. Zywnosc. Technologia, Jakosc 1998, 4, 19-46.

[5] C. J. Tijsen, H. J. Kolk, E. J. Stamhuis, A. A. C. M. Beenackers: An experimental study on the carboxymethylation of granular potato starch in non-aqueous media. Carbohydr. Polym. 2001, 45, 219-226.

[6] T. Heinze, K. Pfeiffer, T. Liebert, U. Heinze: Effective approaches for estimating the functionalization pattern of carboxymethyl starch of different origin. Starch/Stärke 1999, 51, 1116.

[7] D. Bhattacharyya, R. S. Singhal, P. R. Kulkarni: A comparative account of conditions for synthesis of sodium carboxymethyl starch from corn and amaranth starch. Carbohydr. Polym. 1995, 27, 247-253.
[8] Z. Stojanovic, K. Jeremic, S. Jovanovic: Synthesis of carboxymethyl starch. Starch/Stärke 2000, 52, 413-419.

[9] Y. Jie, C. Wen-ren, R. M. Manurung, K. J. Ganzeveld, H. J. Heeres.: Exploratory studies on the carboxymethylation of cassava starch in water-miscible organic media. Starch/ Stärke 2004, 56, 100-107.

[10] D. Montgomery: Response surface methods and designs, in Design and Analysis of Experiments. John Wiley \& Sons, New York, 1991.

[11] L. M. Kooijman, K. J. Ganzeveld, R. M. Manurung, H. J. Heeres: Experimental studies on the carboxymethylation of arrowroot starch in isopropanol-water media. Starch/Stärke 2003, 55, 495-503.

[12] S. Ketsilp, K. Sangseethong, K. Sriroth: Effect of reaction conditions on carboxymethylation of cassava starch, in The $14^{\text {th }}$ Annual Meeting of the Thai Society for Biotechnology, Khon Kaen, Thailand, November 12-15, 2002.

[13] J. Chen, J. Jane: Preparation of granular cold-water-soluble starches by alcoholic-alkaline treatment. Cereal Chem. 1994, 71, 618-622.

[14] S. A. S. Craig, C. C. Maningat, P. A. Seib, R. C. Hoseney: Starch paste clarity. Cereal Chem. 1989, 66, 173-182.

[15] M. I. Khalil, A. Hashem, A. Hebeish: Carboxymethylation of maize starch. Starch/Stärke 1990, 42, 60-63.

[16] J. Chen, J. Jane: Properties of granular cold-water-soluble starches prepared by alcoholic-alkaline treatments. Cereal Chem. 1994, 71, 623-626.

[17] J. Singh, N. Singh: Studies on the morphological and rheological properties of granular cold water soluble corn and potato starches. Food Hydrocoll. 2003, 17, 63-72.

[18] R. L. Whistler, J. R. Daniel: Carbohydrates, in Food Chemistry (Ed. O. R. Fennema) Marcel Dekker, New York, 1985.

[19] C. Clasen, W. M. Kulicke: Determination of viscoelastic and rheo-optical material functions of water-soluble cellulose derivatives. Prog. Polym. Sci. 2001, 26, 1839-1919.

[20] M. R. Jacobson, M. Obanni, J. N. BeMiller: Retrogradation of starches from different botanical sources. Cereal Chem. 1997, 74, 511-518.

(Received: April 21, 2004)

(Revised: October 28, 2004)

(Accepted: October 28, 2004) 